\title{
Salivary Porphyromonas gingivalis predicts outcome in oral squamous cell carcinomas: a cohort study
}

Qingli Chen ${ }^{1}$, Zhe Shao ${ }^{1,2}$, Ke Liu $^{1,2}$, Xiaocheng Zhou ${ }^{1}$, Lin Wang ${ }^{1}$, Erhui Jiang ${ }^{1,2}$, Tingting Luo ${ }^{1}$ and Zhengjun Shang ${ }^{1,2^{*}}$

\begin{abstract}
Background: Studies suggest Porphyromonas gingivalis $(P g)$ increased the incidence of oral squamous cell carcinoma (OSCC). However, fimA genotypes distribution of $\mathrm{Pg}$, the origination of $\mathrm{Pg}$ in tissue, and its prognostic value are inconclusive. We aimed to investigate the frequency of fimA genotypes in OSCC patients, study the association between $\mathrm{Pg}$ and OSCC, and explore the prognostic value of Pg.

Methods: The abundance of $\mathrm{Pg}$ in saliva from the OSCC group and the OSCC-free group was analysed by qPCR. The presence of $\mathrm{Pg}$ was explored in OSCC tissue and para-cancerous tissue by in situ hybridization. The frequency of fimA genotypes in saliva and OSCC tissue was determined by PCR, then PCR products were sequenced and compared. Clinical data were extracted, and patients followed up for a median period of 23 months. Clinicopathological variables were compared with the abundance of $P g$ using Pearson Chi-square test or Fisher's exact test. The disease-free survival (DFS) rate was calculated by Kaplan-Meier method with log-rank tests.

Results: Comparing the OSCC-free group, 95 patients with OSCC showed a high abundance of $\mathrm{Pg}$ in saliva $(P=0.033)$, and OSCC tissue showed strong in situ expression of $\mathrm{Pg}$ compared with paired normal tissue. Patients with OSCC showed a dominant distribution of Pg with genotype I + Ib (21.1\%), II (31.6\%), and IV (21.1\%). FimA genotypes detected in saliva were in accordance with those in OSCC tissue, there was, moreover, a significant similarity in amplified $\mathrm{Pg}$ fragments. Of the 94 responsive OSCC patients, the recurrence rate was 26.6\% (25/94). Overabundance of $P g$ in saliva showed advanced pathologic staging $(P=0.008)$, longer disease-free time $(P=0.029)$ and lower recurrence rate ( $P=0.033)$. The overabundance of $P g$ in saliva was associated with improved disease-free survival $(P=0.049)$.
\end{abstract}

Conclusions: This study indicated that Pg might involve in the pathogenesis of OSCC, Pg carrying fimA I, Ib, II, and IV were prevalent genotypes in patients with OSCC, the provenance of $\mathrm{Pg}$ in OSCC tissue might be from the salivary microbial reservoir, and the abundance of $\mathrm{Pg}$ in saliva might consider as a favorable potential prognostic indicator in OSCC.

Keywords: Porphyromonas gingivalis, FimA, Genotype, Oral squamous cell carcinoma, Fimbriae, Prognosis

*Correspondence: shangzhengjun@whu.edu.cn

${ }^{1}$ The State Key Laboratory Breeding Base of Basic Science of Stomatology (Hubei-MOST) \& Key Laboratory of Oral Biomedicine Ministry of Education, School \& Hospita1 of Stomatology, Wuhan University, 237 Luoyu Road, Wuhan, People's Republic of China

Full list of author information is available at the end of the article

\section{Background}

There were approximately 354,864 new cases of lip and oral cavity cancer and 177,384 related deaths worldwide in 2018 [1]. Oral squamous cell carcinoma (OSCC) is the most common malignant disease in the head and neck besides non-melanoma skin cancer. Traditionally, risk 
factors associated with OSCC included tobacco/alcohol consumption, betel quid chewing, virus infection, dietary factor, vitamin/mineral deficiencies, occupational exposures, and heritable conditions. However, accumulating epidemiologic, clinicopathologic, and molecular studies have proven that oral microbial species played an important role in the carcinogenesis of OSCC [2].

Porphyromonas gingivalis (Pg), an anaerobic Gramnegative bacterium, was associated with a high risk of OSCC in extensive studies [3-9]. However, the effect of $P g$ infection on the prognosis of OSCC is unclear. Recently, $P g$ infection in OSCC tissue showed a trend (HR $0.34 ; P=0.055$ in multivariate regression analyses) towards improved overall survival [10]. Given that salivary microbiota is stable and saliva is easy and noninvasive to collect, saliva is an acceptable biofluid for the evaluation of the oral microbiome [11, 12]. We hypothesized that the abundance of $P g$ in saliva was associated with a favorable prognosis in patients with OSCC.

Fimbriae are crucial to initial attachment, organization of biofilms, and adhesion that mediate invasion and colonization of cells, although $\mathrm{Pg}$ has an arsenal of virulence factors, such as gingipain, lipopolysaccharide, and capsule [13]. The major fimbriae fimA and the minor one mfa1 were two distinct fimbriae expressed in $P g$. Six genotypes (types I-V, Ib) of fimA have been identified based on sequence variations. Previous studies have reported a high prevalence of fimA genotype II in periodontitis patients, and fimA genotype Ib, II and, IV are more aggressive [14-17]. However, no study was found evaluating the frequency of fimA genotypes in patients with OSCC.

In this study, we explored the association between $P g$ and OSCC. Meanwhile, we investigated the prevalence of fimA genotypes in patients with OSCC. To verify the origination of $P g$ in the OSCC tissue, we compared the frequency of fimA genotypes in saliva and those in OSCC tissues. Furthermore, we compared the amplified $P g$ fragments from OSCC tissue and those from saliva. The potential prognostic value of $P g$ in OSCC was also assessed.

\section{Methods}

Approval from the institutional review board was obtained at the Hospital of Stomatology Wuhan University before starting the study (2016-60). Informed consent was obtained from each patient.

The inclusion criterion was patients with primary OSCC managed by curative-intent, and a total number of 111 consecutive patients were included from October 2018 and April 2019. The exclusion criteria were patients: (1) received oral prophylaxis in the latest three months (one patient), (2) underwent radiotherapy and/ or chemotherapy before surgery (three patients), (3) edentulous (two patients), (4) refused to receive surgery, (5) disagreed to participate in this study (four patients disagreed to the collection of saliva and six patients did not fast overnight before saliva sample collection).

A total number of 95 patients with OSCC (65 male and 30 female subjects, aged $21-82$ years, mean age 55.8) treated in the hospital were included in this study. Pathological staging was stratified in accordance with the eighth edition of the American Joint Committee on Cancer [18]. Except for one patient with bone metastatic OSCC, all patients were M0 category. The control group comprised 39 OSCC-free subjects (21 males and 18 females, aged 33-76 years, mean age 52.6) diagnosed with salivary gland disease, lymphadenopathy, lymphoma, buccal or tongue chronic infection, epulis, ranula, lipoma, lymphoepithelial cyst, and sebaceous gland carcinoma.

Clinical records were retrieved. Assessed clinicopathological variables included age, gender, systematic disease, location of the tumor, size, pathological report, smoking, alcohol consumption, and treatment. Systemic disease included hypertension, diabetes, coronary artery disease, and chronic hepatitis B. Sixty-four patients underwent surgery, twenty-two patients underwent surgery+ radiotherapy (IMRT: 76 Grays to 63 Grays) and eight patients underwent surgery+chemo-radiotherapy (Docetaxel, Cisplatin, 5-Fluorouracil). Pathological diagnosis was established by one pathologist and confirmed by another experienced pathologist from the department of pathological at the Hospital of Stomatology Wuhan University.

Patients were followed up from discharge by telephone or clinical assessment. Pathologic confirmation of recurrence was obtained in patients with clinical signs or symptoms. Disease-free survival (DFS) is defined as the time (in months) from the date of discharge to March 2021 or until the date recurrence was diagnosed.

A total of 134 saliva samples were collected between 6 a.m. and 8 a.m. following an overnight fast and refrainment of tooth brushing. Subjects were asked to swish vigorously with $40 \mathrm{~mL}$ sterilized double distilled water (bacteria negative in PCR assay) for $1 \mathrm{~min}$, and then to expectorate into another specimen tube [19]. The saliva samples were centrifuged at 14,000 rpm for $15 \mathrm{~min}$, and then the cell pellet was suspended in $1 \mathrm{~mL}$ of sterile TE buffer. Saliva samples stored at $-80^{\circ} \mathrm{C}$ until testing.

Bacterial DNA was extracted from saliva samples using a commercial DNA extraction kit (DP302, Tiangen, China) according to the manufacturer's protocol, except adding an enzymatic lysis step with lysozyme $(20 \mathrm{mg} / \mathrm{ml}$, $\left.37^{\circ} \mathrm{C}, 60 \mathrm{~min}\right)$. The resultant DNA was stored at $-20^{\circ} \mathrm{C}$ until in PCR. 
A total of 15 out of 95 fresh-frozen OSCC tissue samples were obtained. DNA was extracted using the Total DNA/RNA/Protein Kit (R6734, Omega Bio-tek, USA) according to the procedure recommended by the manufacturer. Quantification of $P g$ in saliva samples and detection of fimA genotypes were measured by Realtime quantitative PCR. Amplifications were performed in duplicate on Bio-Rad CFX96 thermal cycler (Bio-Rad Laboratories, USA). The primers, synthesized by Sangon Biotech (Shanghai, China), were listed in Table 1.

The reaction mixture of $20 \mu \mathrm{L}$ was composed of $50 \mathrm{ng}$ saliva DNA template or $2 \mu \mathrm{L}$ tissue DNA template, $0.4 \mu \mathrm{M}$ of the specific primers, ChamQTM SYBR ${ }^{\circledR} \mathrm{qPCR}$ Master with a final concentration of 1X (Q311, Vazyme, China), and an appropriate dose of sterilized DNaseRNase-free water. The conditions for Real-time quantitative PCR were as follows: $94^{\circ} \mathrm{C}$ for $5 \mathrm{~min}$, then 28 cycles for $P g$ or 40 cycles for fimA genotypes of $30 \mathrm{~s}$ at $94{ }^{\circ} \mathrm{C}$, $45 \mathrm{~s}$ at $58{ }^{\circ} \mathrm{C}$ or $60{ }^{\circ} \mathrm{C}$, and $1 \mathrm{~min}$ at $72{ }^{\circ} \mathrm{C}$; with a final extension of $10 \mathrm{~min}$ at $72^{\circ} \mathrm{C}$. Melting curves were generated from $60{ }^{\circ} \mathrm{C}$ to $95^{\circ} \mathrm{C}$ and read every $0.5^{\circ} \mathrm{C}$ for $5 \mathrm{~s}$. An average $\mathrm{Ct}$ value was obtained. The $\Delta \mathrm{Ct}$ for $P g$ was determined by subtracting the Ct value of $P g$ from that of universal primer. The relative abundance of $\mathrm{Pg}$ was calculated by the $2^{-\Delta \Delta \mathrm{Ct}}$ method.

Amplified PCR products of fimA genotype from Realtime quantitative PCR were checked on $2 \%$ agarose gel (ST004L, Beyotime, China). This was done using 1X Tris Acetate-EDTA buffer (TAE) from 50X TAE (ST716, Beyotime, China). Gels were stained with $4 \mathrm{~S}$ GelRed
(A616697, Sangon Biotech, China). Image results were captured with the digital imaging system (NuGenius, SYNGENE, UK). One pair of amplified $P g$ fragments from OSCC tissue and saliva were confirmed following nucleotide sequencing by Sangon Biotech (Shanghai, China) and the correlation of two sequences by aligning two sequences with BLAST (http://www.ncbi.nlm.nih. gov/BLAST) [23].

Among 15 OSCC tissue patients, remaining OSCC tissue and normal tissue adjacent to OSCC from one patient were fixed in $4 \%$ paraformaldehyde, paraffinembedded, and cut into $4 \mu \mathrm{m}$ sections, which was stained with haematoxylin and eosin, gram and subjected to in situ hybridization (ISH) using Enhanced Sensitive ISH Detection kit I (POD) (MK1030, Boster, China) according to the manufacturer's instructions. The probe is listed in Table 1. Omission of the probe was obtained as the negative controls.

\section{Statistical analysis}

Shapiro-Wilk test was used to assess whether or not data were normally distributed. Normally distributed data were analysed by Student's t test and presented as Mean \pm Standard Deviation. The data without normal distribution presented as the median and inter-quartile range $(\mathrm{M}, \mathrm{Q})$ and analysed by the Mann-Whitney $\mathrm{U}$ test. Categorical variables were analysed by Pearson Chi-square test or Fisher's exact test. The cutoff point to convert the number of $P g 16 \mathrm{~S}$ rRNA gene copies into categorical data (low, $<4$ and high, $\geq 4$ ) was performed using

Table 1 Specific oligonucleotides used in this study

\begin{tabular}{|c|c|c|c|}
\hline Primer & Sequence $\left(5^{\prime}-3^{\prime}\right)$ & Annealing temperature $\left({ }^{\circ} \mathrm{C}\right)$ & References \\
\hline \multirow[t]{2}{*}{ Universal primers } & F:TCCTACGGGAGGCAGCAGT & 60 & {$[20]$} \\
\hline & R: GGACTACCAGGGTATCTAATCCTGTT & & \\
\hline \multirow[t]{2}{*}{$P g$} & F: ACCTTACCCGGGATTGAAATG & 58 & {$[20]$} \\
\hline & R: CAACCATGCAGCACCTACATAGAA & & \\
\hline \multirow[t]{2}{*}{ fimA I } & F: CTGTGTGTTTATGGCAAACTTC & 58 & {$[21]$} \\
\hline & R: AACCCCGCTCCCTGTATTCCGA & & \\
\hline \multirow[t]{2}{*}{ fimA Ib } & F: CAGCAGAGCCAAAAACAATCG & 58 & {$[14]$} \\
\hline & R:TGTCAGATAATTAGCGTCTGC & & \\
\hline \multirow[t]{2}{*}{ fimA ॥ } & F: GCATGATGGTACTCCTTTGA & 58 & {$[22]$} \\
\hline & R: CTGACCAACGAGAACCCACT & & \\
\hline \multirow[t]{2}{*}{ fimA III } & F: ATTACACCTACACAGGTGAGGC & 58 & {$[21]$} \\
\hline & R: AACCCCGCTCCCTGTATTCCGA & & \\
\hline \multirow[t]{2}{*}{ fimA IV } & F: CTATTCAGGTGCTATTACCCAA & 58 & {$[21]$} \\
\hline & R: AACCCCGCTCCCTGTATTCCGA & & \\
\hline \multirow[t]{2}{*}{ fimA $\vee$} & F: AACAACAGTCTCCTTGACAGTG & 58 & {$[14]$} \\
\hline & R:TATTGGGGGTCGAACGTTACTGTC & & \\
\hline Pg probe used in ISH & CAATACTCGTATCGCCCGTTATTC-Digoxin & & {$[3]$} \\
\hline
\end{tabular}

Pg: Porphyromonas gingivalis. ISH: In situ hybridization 
X-tile software [24]. The Kaplan-Meier log-rank test was performed to compare the DFS. All two-tailed $P$ values $<0.05$ were considered significant. All analyses were carried out using IBM SPSS Statistics software (IBM SPSS Statistics V.25.0, USA).

\section{Results}

As showed in Table 2, compared with controls matched for gender and age $(P>0.05$, respectively), OSCC patients showed an overabundance of $P g$ in saliva $(P<0.05)$. To exclude contamination of samples, $P g$ was also detected in tissues by ISH from one patient.

Table 2 Overabundance of $\mathrm{Pg}$ in saliva from OSCC patient

\begin{tabular}{llcc}
\hline & \multicolumn{2}{l}{ Porphyromonas gingivalis } & $P$ \\
\cline { 2 - 3 } & Low & High & \\
\hline OSCC & 84 & 11 & 0.033 \\
OSCC-free & 39 & 0 & \\
\hline
\end{tabular}

Compared with normal tissue which is adjacent to OSCC, OSCC tissue showed strong in situ expression of $P g$ (Fig. 1).

To evaluate the frequency of different fimA genotypes in patients with OSCC. Amplified PCR products of $f i m A$ genotype were checked on $2 \%$ agarose gel electrophoresis. The distribution of fimA genotype from the saliva of 95 OSCC patients was listed in Table 3. FimA genotype I and Ib were detected in $20(21.1 \%)$ specimens, genotype II in 30 (31.6\%) specimens, genotype III in $4 .(4.2 \%)$ specimens, genotype IV in $20(21.1 \%)$ specimens, genotype $\mathrm{V}$ in $2(2.1 \%)$ specimens. We also found two or more genotypes of fimA from one sample. FimA genotype I, Ib, and II was detected in $1(1.1 \%)$ participant, genotype I, Ib, and III in $2(2.1 \%)$ participants, genotype I, Ib and IV in 1 (1.1\%) participant, genotype $\mathrm{I}$, Ib, and $\mathrm{V}$ in 1 (1.1\%) participant, genotype II and IV in $3(3.2 \%)$ participants, genotype I, Ib, II, and IV in 1 (1.1\%) participant. Ten participants showed negative on $2 \%$ agarose gel electrophoresis assay. This finding supported the dominant distribution of $P g$ with genotype I, Ib, II, and IV in saliva from OSCC patients.

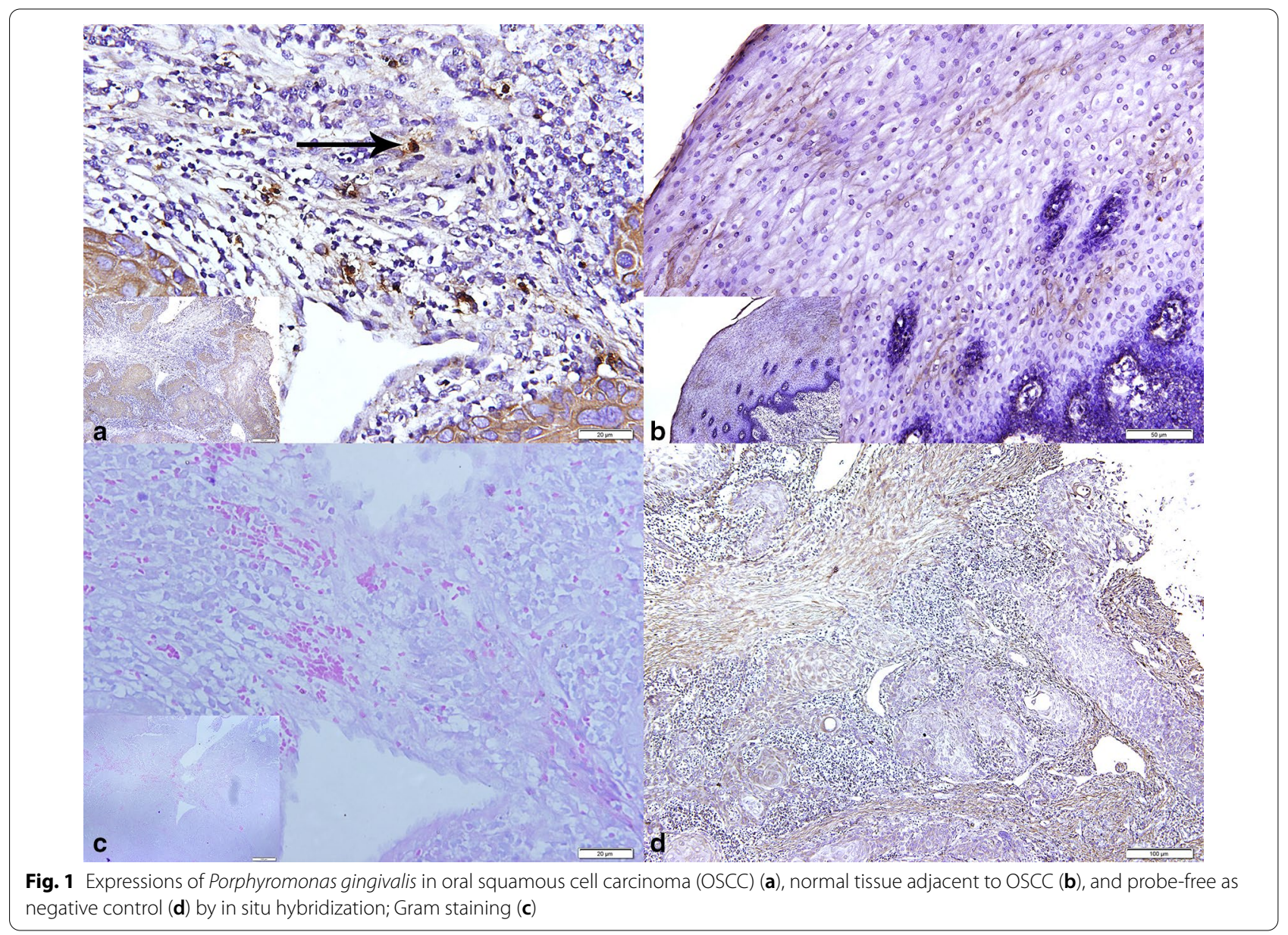


Table 3 Clinicopathological details

\begin{tabular}{|c|c|c|c|c|c|}
\hline \multirow[t]{2}{*}{ Parameters } & & \multirow[t]{2}{*}{ Number (\%) } & \multicolumn{2}{|c|}{ Porphyromonas gingivalis } & \multirow[t]{2}{*}{$P$ value } \\
\hline & & & Low & High & \\
\hline Age (years) & $55.8 \pm 12.7$ & $95(100)$ & $55.7 \pm 12.6$ & $56.5 \pm 14.6$ & 0.860 \\
\hline \multirow[t]{2}{*}{ Gender } & Male & $65(68.4)$ & 57 & 8 & 0.999 \\
\hline & Female & $30(31.6)$ & 27 & 3 & \\
\hline \multirow[t]{2}{*}{ Systemic disease } & No & $69(72.6)$ & 61 & 8 & 0.999 \\
\hline & Yes & $26(27.4)$ & 23 & 3 & \\
\hline \multirow[t]{2}{*}{ Pathologic staging } & pStage I+ II & $69(72.6)$ & 65 & 4 & 0.008 \\
\hline & pStage III + IV & $26(27.4)$ & 19 & 7 & \\
\hline \multirow[t]{2}{*}{ Smoking } & No & $45(47.4)$ & 39 & 6 & 0.612 \\
\hline & Yes & $50(52.6)$ & 45 & 5 & \\
\hline \multirow[t]{2}{*}{ Alcohol consumption } & No & $57(60.0)$ & 50 & 7 & 0.999 \\
\hline & Yes & $38(40.0)$ & 34 & 4 & \\
\hline \multirow[t]{5}{*}{ Location } & Buccal & $21(24.2)$ & 21 & 2 & 0.447 \\
\hline & Tongue & $52(54.7)$ & 47 & 5 & \\
\hline & Gingiva & $11(11.6)$ & 9 & 2 & \\
\hline & Floor of mouth & $5(5.3)$ & 4 & 1 & \\
\hline & Hard palate & $4(4.2)$ & 3 & 1 & \\
\hline \multirow[t]{3}{*}{ Differentiation grade } & Well & $17(19.1)$ & 16 & 1 & 0.735 \\
\hline & Moderate & $65(73.0)$ & 57 & 8 & \\
\hline & Poor & $7(7.9)$ & 6 & 1 & \\
\hline \multirow[t]{12}{*}{ FimA genotypes } & $I+I b$ & $20(21.1)$ & 17 & 3 & $0.795^{*}$ \\
\hline & $\|$ & $30(31.6)$ & 27 & 3 & \\
\hline & III & $4(4.2)$ & 4 & 0 & \\
\hline & IV & $20(21.1)$ & 16 & 4 & \\
\hline & V & $2(2.1)$ & 2 & 0 & \\
\hline & $1+\mid b+11$ & $1(1.1)$ & 1 & 0 & \\
\hline & $1+|b+|||$ & $2(2.1)$ & 2 & 0 & \\
\hline & $I+I b+I V$ & $1(1.1)$ & 1 & 0 & \\
\hline & $I+I b+V$ & $1(1.1)$ & 1 & 0 & \\
\hline & $I I+I V$ & $3(3.2)$ & 3 & 0 & \\
\hline & $I+I b+I I+I V$ & $1(1.1)$ & 1 & 0 & \\
\hline & Untyped & $10(10.5)$ & 9 & 1 & \\
\hline \multirow[t]{2}{*}{ Treatment $^{\dagger}$} & Surgery & $64(68.1)$ & 60 & 4 & - \\
\hline & Surgery + RT or CRT ** & $30(31.9)$ & 23 & 7 & \\
\hline \multirow[t]{2}{*}{ Outcome $^{t}$} & Recurrence & $24(25.5)$ & 25 & 0 & 0.033 \\
\hline & Disease-free & $70(74.5)$ & 58 & 11 & \\
\hline Disease-free time (months) & 23,10 & $94(98.9)$ & $22,13.8$ & 27,8 & 0.029 \\
\hline
\end{tabular}

*Chi-square test was used among fimA genotype I+ Ib, II, III, IV, V

${ }^{\dagger}$ One patient dropped out in follow-up

**RT: Radiotherapy, CRT: Chemo-radiotherapy

To clarify the homogeneity of $P g$ between saliva and OSCC tissue, the frequency of fimA genotypes was also detected in fifteen OSCC tissues. Among fifteen patients, the fimA genotypes detected in saliva were in accordance with those in OSCC tissue (Table 4). Besides, amplified $P g$ fragments from OSCC tissue and those from saliva were examined in one patient, we found a significant correlation in nucleotide similarity (Figs. 2, 3). Collectively, these results implied that $P g$ in OSCC tissue might originate from the salivary microbial reservoir.

The deadline for follow-up was March 2021. After a median follow-up period of 23 months (range 3 to 28 months). Ninety-four patients were available for the follow-up visit, but one patient was non-responsive to 
Table 4 The frequency of fimA genotypes in saliva and in oral squamous cell carcinoma tissues

\begin{tabular}{lllllll}
\hline & I+lb & II & III & IV & V & I+ Ib+ III \\
\hline Saliva & 3 & 6 & 1 & 2 & 2 & 1 \\
Tissue & 3 & 6 & 1 & 2 & 2 & 1 \\
\hline
\end{tabular}

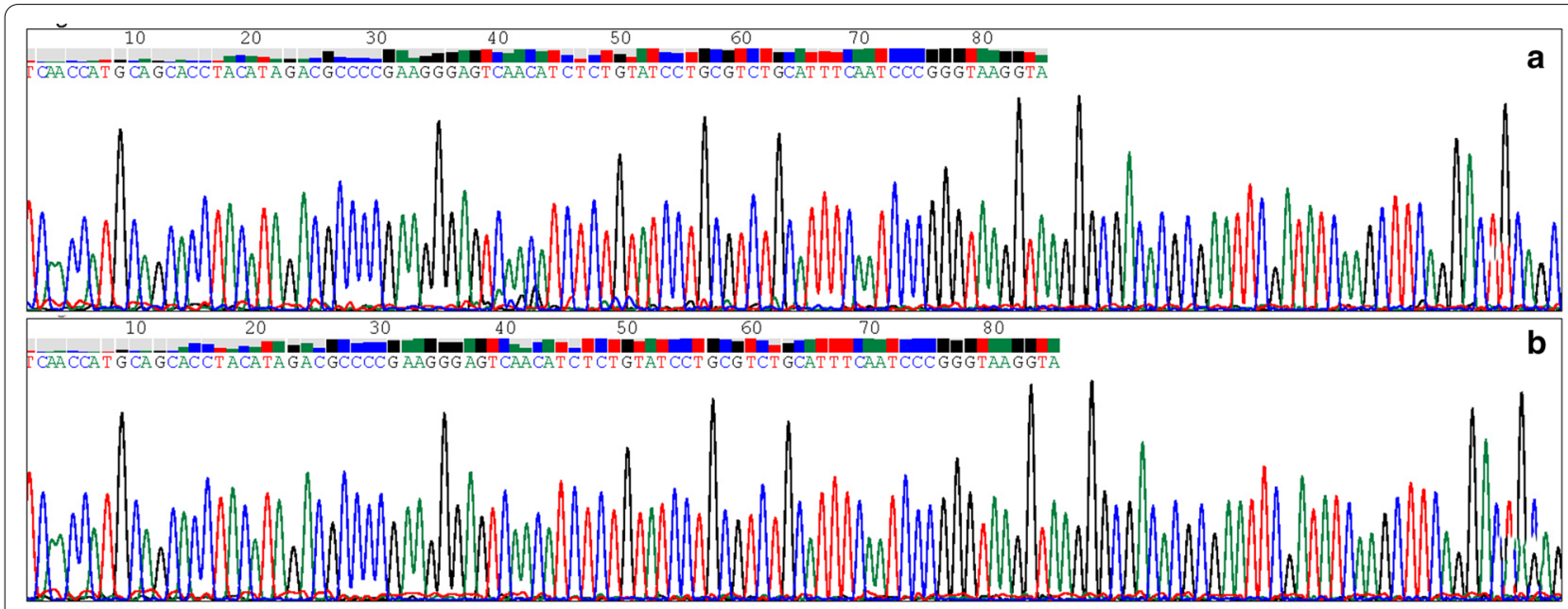

Fig. 2 The PCR product examined Porphyromonas gingivalis in saliva (a) and oral squamous cell carcinoma tissue (b)

\begin{tabular}{|c|c|c|c|c|}
\hline $\begin{array}{l}\text { Score } \\
154 \text { bits(170) }\end{array}$ & $\begin{array}{l}\text { Expect } \\
2 e-43\end{array}$ & $\begin{array}{l}\text { Identities } \\
85 / 85(100 \%)\end{array}$ & $\begin{array}{l}\text { Gaps } \\
0 / 85(0 \%)\end{array}$ & $\begin{array}{l}\text { Strand } \\
\text { Plus/Plus }\end{array}$ \\
\hline Query 11 & $\begin{array}{l}\text { ACCTACATA } \\
\|\|\|\|\end{array}$ & $\begin{array}{l}\text { CCCGAHGGGAGTCAHC } \\
\text { IIIIIIIIIIIII }\end{array}$ & $\begin{array}{l}\text { TCCTGCGT } 60 \\
\text { IIIIII }\end{array}$ & \\
\hline Sbjet 1 TChid & ACCTACATA & CCCGAHGGGAGTCALC & TCCTGCGT 6 & \\
\hline Query 61 CTGC & CCGGGTAAG & 85 & & \\
\hline $\begin{array}{l}\text { Sbjet } 61 \text { CTGC } \\
\text { Fig. } 3 \text { The homology }\end{array}$ & CCGGGTARG & $\begin{array}{l}85 \\
\text { detected in saliva and } 0\end{array}$ & ell carcinoma & \\
\hline
\end{tabular}

any form of contact. Recurrence was diagnosed as the endpoint for 25 patients, with a $26.6 \%(25 / 94)$ cumulative recurrence rate (Fig. 4).

Clinicopathological information of OSCC patients is shown in Table 3. The distribution of clinicopathological outcomes was compared with the abundance of $P g$ to assess the potential prognostic variables. Patients with the overabundance of $P g$ in saliva had an advanced pathologic staging than those with a low abundance of $P g(P=0.008)$. While, compared with the weak group, patients with the overabundance of $P g$ in saliva had a longer disease-free time $(Z=-2.188, P=0.029)$. The overabundance of $P g$ in saliva was associated with a lower recurrence rate $(P=0.033)$. However, differences were not statistically significant by age, gender, systemic disease, smoking, alcoholic consumption, location, differentiation grade, and fimA genotypes. Neither single fimA genotype was statistically significantly associated with any clinicopathological parameters.

A total of 94 cases with follow-up data were included in the survival analysis. Univariate analysis showed that the overabundance of $P g$ was a favorable prognostic factor $($ Chi-square $=3.86, P<0.05)$ (Fig. 5). Statistically, age, gender, FimA genotypes, systemic disease, pathologic staging, smoking, alcoholic consumption, location, differentiation grade, and treatment were not the independent 

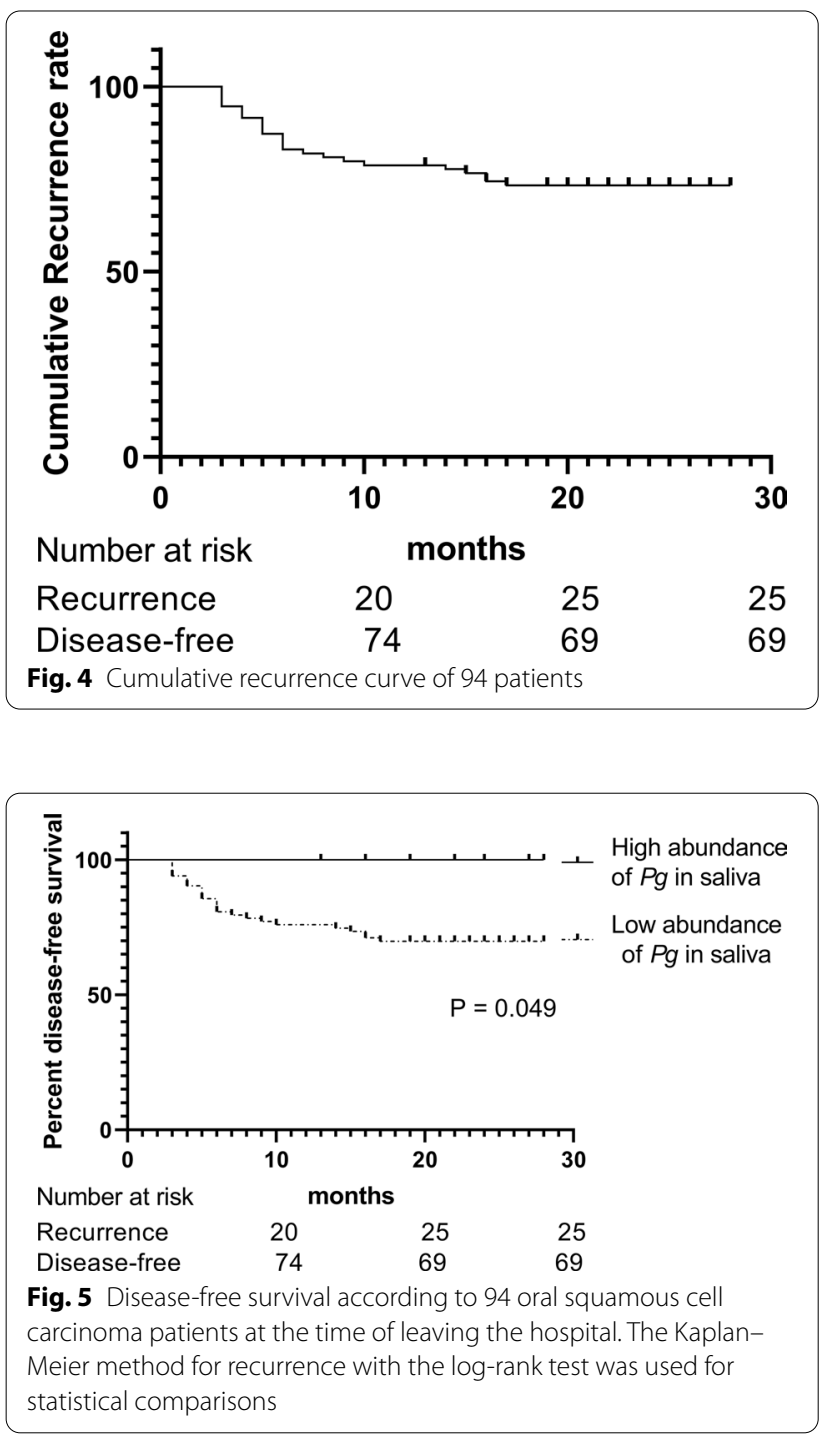

prognostic indicators. Consequently, our results showed the overabundance of $P g$ was associated with favorable outcomes in patients with OSCC.

\section{Discussions}

With recent breakthroughs in high-throughput geneticbased tools, there has been a hot issue concerning the relationship between the oral microbiome and neoplasms, especially OSCC. Recently accumulating evidence indicated the relationship between $P g$ and OSCC. Immortalized oral keratinocytes stimulated with $P g$ led to a more aggressive malignant profile phenotype and contributing to enhanced tumor features [25]. The serum immunoglobulin $\mathrm{G}$ antibody against $\mathrm{Pg}$ was higher in OSCC patients compared with non-OSCC patients [4]. $P g$ increased the size and the multiplicity of carcinoma to promote the development of oral cancer [5]. Previous studies have confirmed the association between $P g$ and OSCC by examining the abundance of $P g$ in the saliva of patients and unveiled that patients with medium and poor differentiation, overall clinical stage III and stage IV, lymph node metastasis, and shorter overall survival associated with $P g$ involvement $[3,10]$. But in multivariate analyses, colonization of $P g$ was a favorable prognostic factor, with a strong tendency towards statistical significance (HR 0.340, 95\% CI 0.112-1.025, $P=0.055$ ) [10]. Our study also verified the overabundance of $P g$ from OSCC patients compared with OSCC-free subjects in saliva, and the overabundance of $P g$ in saliva was more likely to correspond with the advanced pathological stage. Those results are in resonance to previous reports [3]. To exclude contamination of samples, we examined the presence of $\mathrm{Pg}$ in OSCC tissue by ISH. Consistent with the previous report [3], there was high enrichment of $P g$ in OSCC tissue compared with normal tissue adjacent to OSCC. After a median follow-up period of 23 months, the OSCC recurred in $26.6 \%$ (25/94) of patients. As the same as our findings, it was reported early-stage patients have a $90-95 \%$ survival rate for one year or more, and advanced-stage patients have a $65-70 \%$ survival rate [26].

In this study, compared with the weak group, patients with the overabundance of $P g$ in saliva had a longer disease-free time and lower recurrence rate. Those suggested that $P g$ may affect the prognosis of OSCC. Most interestingly, we found the overabundance of $P g$ is a favorable prognostic factor for DFS. Contrary to popular belief, they found that $P g$ was associated with a higher risk of pancreatic cancer [27, 28], esophageal squamous cell carcinoma $[29,30]$, and oral squamous cell carcinoma [3, 10]. Meanwhile, $P g$ was associated with poor overall survival rates in esophageal squamous cell carcinoma [31]. Furthermore, patients with a high level of $P g$ had the worst prognosis in esophageal squamous cell carcinoma [30]. Besides the population and follow-up period contributed to this prognostic incongruity, the inherent mechanistic also should be taken into consideration.

One of the most vital virulence in $P g$ has been supposed to the presence of fimbriae, which plays an important role in adhesion, colonization, and invasion to tissues [32]. Most of the studies focused on the distribution of fimA genotypes in periodontitis. However, the frequency of fimA genotypes in OSCC was not clear. FimA genotypes I and Ib could be discriminated by Rsa I enzyme digestion. However, discrimination of genotypes I and Ib seems to be improbable [14]. Besides, there were no differences in the immunological analysis between fimA I and Ib fimbriae [14]. So we consider fimA genotypes I and $\mathrm{Ib}$ as a whole. 
In this study, the association of fimA genotypes and clinicopathological parameters was not statistically significant. However, the predominantly detected fimA genotypes in OSCC were genotypes I, Ib, II, and IV. Several studies concluded that nucleotide genetic variation was likely associated with virulence. Some reported fimA genotypes Ib, II, and IV are the most virulent fimbriae in periodontitis and assist in adhesion and invasion [14,33]. It was reported that fimA genotype Ib, II, and IV led to more severe infections and inflammations [34, 35]. Clinical isolation of $P g$ from chronic periodontitis patients also supported the virulence of fimA genotypes Ib, II, and IV [36]. Different $P g$ fimA genotype was injected subcutaneously, and Nakano et al. found that the weakest inflammatory response was induced by genotype III [35]. FimA genotype V was the least amount of genotypes in this study. The reason might be the low prevalence $(0-29 \%)$ of this genotype [37]. Single $P g$ fimA genotype was determined by more than $70 \%$ of OSCC patients, and two or more genotypes were also detected in a subset of the subjects. Approximately $10 \%$ of the samples were multiple genotypes in this population, which was less than the results of other studies [14, 17]. Researchers attributed to the limitations of PCR in the discrimination of fimA genotypes and the possibility of classifying new genotypes [38].

Due to the conservative properties of DNA, the bacterial $16 \mathrm{~S}$ ribosomal DNA allows identification of the genus and species. Analysis of $P g$ nucleotide sequences in the OSCC tissue and those in the saliva showed a homology of $100 \%$. Moreover, the distribution of fimA genotypes in OSCC tissue is according to those in saliva. Those results support the origination of $P g$ in OSCC tissues might be from the salivary microbial reservoir [39].

The limitations of this study included the short followup period and small sample. Further research will need to be done to elucidate the mechanism of the prognostic role. Except for fimbriae of $P g$, important virulence included: encapsulation (K1-K6), gingipains (types $A, B, C$ ) as well as lysine-specific types I and II, may also involve in the carcinogenesis of OSCC. Those remain to be uncovered in future studies.

\section{Conclusions}

This study found the overabundance of $P g$ was associated with OSCC, and patients with advanced pathological stage, longer disease-free time, and the lower recurrence rate were related to the overabundance of $P g$. Meanwhile, the overabundance of $P g$ was a favorable prognostic factor in patients with OSCC. Furthermore, there was a dominant distribution of $\mathrm{Pg}$ with genotype I, Ib, II, and IV from patients with OSCC, and the origination of $P g$ in the tumor might be from the salivary microbial reservoir.
Abbreviations

OSCC: Oral squamous cell carcinoma; Pg: Porphyromonas gingivalis; ISH: In situ hybridization.

\section{Acknowledgements}

Thanks to all staff in our laboratory for their kind heart and selfless contribution as well as Pro. Zhongcheng Gong (Oncological Department of Oral and Maxillofacial Surgery, The First Affiliated Hospital of Xinjiang Medical University) for his encouragement.

\section{Authors' contributions}

QC, Z Shao, Z Shang conceived the study; QC, KL performed the experiments; $X Z$, LW performed the statistical analysis; QC and Z Shao wrote the initial draft of the manuscript; LT, EJ revised and edited the manuscript; Z Shang finalised the manuscript; All authors read and approved the final manuscript.

\section{Funding}

This study was supported by the National Natural Science Foundation of China (Grant 81672666 to Zhengjun Shang). The funding organization was not involved in the design of the study, the collection, analysis, and interpretation of data, or in writing the manuscript.

\section{Availability of data and materials}

The raw data are confidential and cannot readily be shared. Researchers need to obtain permission from the Institutional Review Board and apply for access to the data from The Ethics Committee of Stomatological Hospital, Wuhan University.

\section{Declarations}

Ethics approval and consent to participate

Approval from the institutional review board was obtained at the Hospital of Stomatology Wuhan University before starting the study (2016-60). Informed consent was written by each patient. Animal Ethics clearance is not applicable, as this study does not involve any animals.

\section{Consent for publication}

Not applicable.

\section{Competing interests}

The authors declare that they have no competing interests.

\section{Author details}

${ }^{1}$ The State Key Laboratory Breeding Base of Basic Science of Stomatology (Hubei-MOST) \& Key Laboratory of Oral Biomedicine Ministry of Education, School \& Hospita1 of Stomatology, Wuhan University, 237 Luoyu Road, Wuhan, People's Republic of China. ${ }^{2}$ Department of Oral and Maxillofacial-Head and Neck Oncology, School and Hospital of Stomatology, Wuhan University, Wuhan, People's Republic of China.

Received: 22 July 2020 Accepted: 21 April 2021

Published online: 03 May 2021

\section{References}

1. Bray F, Ferlay J, Soerjomataram I, Siegel RL, Torre LA, Jemal A. Global cancer statistics 2018: GLOBOCAN estimates of incidence and mortality worldwide for 36 cancers in 185 countries. CA Cancer J Clin. 2018;68(6):394-424

2. Karpiński TM. Role of oral microbiota in cancer development. Microorganisms. 2019;7(1):E20.

3. Chang C, Geng F, Shi X, Li Y, Zhang X, Zhao X, et al. The prevalence rate of periodontal pathogens and its association with oral squamous cell carcinoma. Appl Microbiol Biotechnol. 2019;103(3):1393-404.

4. Park DG, Woo BH, Lee BJ, Yoon S, Cho Y, Kim YD, et al. Serum levels of interleukin- 6 and titers of antibodies against Porphyromonas gingivalis could be potential biomarkers for the diagnosis of oral squamous cell carcinoma. Int J Mol Sci. 2019;20(11):E2749. 
5. Wu JS, Zheng M, Zhang M, Pang X, Li L, Wang SS, et al. Porphyromonas gingivalis Promotes 4-nitroquinoline-1-oxide-induced oral carcinogenesis with an alteration of fatty acid metabolism. Front Microbiol. 2018;9:2081.

6. Utispan K, Pugdee K, Koontongkaew S. Porphyromonas gingivalis, lipopolysaccharide-induced macrophages modulate proliferation and invasion of head and neck cancer cell lines. Biomed Pharmacother. 2018:101:988-95.

7. Groeger S, Jarzina F, Domann E, Meyle J. Porphyromonas gingivalis activates NFKB and MAPK pathways in human oral epithelial cells. BMC Immunol. 2017;18(1):1.

8. Woo BH, Kim DJ, Choi JI, Kim SJ, Park BS, Song JM, et al. Oral cancer cells sustainedly infected with Porphyromonas gingivalis exhibit resistance to Taxol and have higher metastatic potentia. Oncotarget. 2017;8(29):46981-92.

9. Ha NH, Woo BH, Kim DJ, Ha ES, Choi Jl, Kim SJ, et al. Prolonged and repetitive exposure to Porphyromonas gingivalis increases aggressiveness of oral cancer cells by promoting acquisition of cancer stem cell properties. Tumour Biol. 2015:36(12):9947-60.

10. Wen L, Mu W, Lu H, Wang X, Fang J, Jia Y, et al. Porphyromonas gingivalis promotes oral squamous cell carcinoma progression in an immune microenvironment. J Dent Res. 2020;99(6):666-75.

11. Rasiah IA, Wong L, Anderson SA, Sissons CH. Variation in bacterial DGGE patterns from human saliva: over time, between individuals and in corresponding dental plaque microcosms. Arch Oral Biol. 2005;50(9):779-87.

12. Belstrøm D, Holmstrup P, Bardow A, Kokaras A, Fiehn NE, Paster BJ. Temporal stability of the salivary microbiota in oral health. PLOS ONE. 2016:11(1):e0147472.

13. Abreu MGL, Kawamoto D, Mayer MPA, Pascoal VDB, Caiaffa KS, Zuza EP, et al. Frequency of Porphyromonas gingivalis fimA in smokers and nonsmokers after periodontal therapy. J Appl Oral Sci. 2019;27:e20180205.

14. Ayala-Herrera JL, Abud-Mendoza C, Gonzalez-Amaro RF, Espinosa-Cristobal LF, Martínez-Martínez RE. Distribution of Porphyromonas gingivalis fimA genotypes in patients affected by rheumatoid arthritis and periodontitis. Acta Odontol Scand. 2018;76(7):520-4.

15. Nagano K, Hasegawa Y, Abiko Y, Yoshida Y, Murakami Y, Yoshimura F. Porphyromonas gingivalis FimA fimbriae: fimbrial assembly by fimA alone in the fim gene cluster and differential antigenicity among fimA genotypes. PLOS ONE. 2012;7(9):e43722.

16. Miura M, Hamachi T, Fujise O, Maeda K. The prevalence and pathogenic differences of Porphyromonas gingivalis fimA genotypes in patients with aggressive periodontitis. J Periodontal Res. 2010;40(2):147-52.

17. Amano A, Kuboniwa M, Nakagawa I, Akiyama S, Morisaki I, Hamada S. Prevalence of specific genotypes of Porphyromonas gingivalis fimA and periodontal health status. J Dent Res. 2000;79(9):1664-8.

18. American Joint Committee on Cancer. Manual for Staging of Cancer 8th ed. New York: Springer International Publishing; 2017:563-588.

19. Fan X, Peters BA, Min D, Ahn J, Hayes RB. Comparison of the oral microbiome in mouthwash and whole saliva samples. PLOS ONE. 2018;13(4):e0194729.

20. Al-Rawi N, Al-Marzooq F. The relation between periodontopathogenic bacterial levels and resistin in the saliva of obese type 2 diabetic patients. J Diabetes Res. 2017;2017:2643079.

21. Amano A, Nakagawa I, Kataoka K, Morisaki I, Hamada S. Distribution of Porphyromonas gingivalis strains with fimA genotypes in periodontitis patients. J Clin Microbiol. 1999:37(5):1426-30.

22. Moon JH, Shin Sl, Chung JH, Lee SW, Amano A, Lee JY. Development and evaluation of new primers for PCR-based identification of type II fimA of Porphyromonas gingivalis. FEMS Immunol Med Microbiol. 2012;64(3):425-8.

23. Altschul SF, Gish W, Miller W, Myers EW, Lipman DJ. Basic local alignment search tool. J Mol Biol. 1990;215(3):403-10.
24. Camp RL, Dolled-Filhart M, Rimm DL. X-Tile: a new bio-informatics tool for biomarker assessment and outcome-based cut-point optimization. Clin Cancer Res. 2004;10(21):7252-9.

25. Hoppe T, Kraus D, Probstmeier R, Jepsen S, Winter J. Stimulation with Porphyromonas gingivalis enhances malignancy and initiates anoikis resistance in immortalized oral keratinocytes. J Cell Physiol. 2019;234(12):21903-14

26. Chaturvedi P, Singh A, Chien CY, Warnakulasuriya S. Tobacco related oral cancer. BMJ. 2019;5(365):12142.

27. Fan X, Alekseyenko AV, Wu J, et al. Human oral microbiome and prospective risk for pancreatic cancer: a population-based nested case-control study. Gut. 2018;67(1):120-7.

28. Fan X, Alekseyenko AV, Wu J, Peters BA, Jacobs EJ, Gapstur SM, et al. Plasma antibodies to oral bacteria and risk of pancreatic cancer in a large European prospective cohort study. Gut. 2013;62(12):1764-70.

29. Peters BA, Wu J, Pei Z, Yang L, Purdue MP, Freedman ND, et al. Oral Microbiome composition reflects prospective risk for esophageal cancers. Cancer Res. 2017;77(23):6777-87.

30. Gao SG, Yang JQ, Ma ZK, Yuan X, Zhao C, Wang GC, et al. Preoperative serum immunoglobulin $\mathrm{G}$ and $\mathrm{A}$ antibodies to Porphyromonas gingivalis are potential serum biomarkers for the diagnosis and prognosis of esophageal squamous cell carcinoma. BMC Cancer. 2018;18(1):17.

31. Gao S, Li S, Ma Z, Liang S, Shan T, Zhang M, et al. Presence of Porphyromonas gingivalis in esophagus and its association with the clinicopathological characteristics and survival in patients with esophageal cancer. Infect Agent Cancer. 2016;11:3.

32. Lin $\mathrm{X}, \mathrm{Wu} \mathrm{J}, \mathrm{Xie} \mathrm{H}$. Porphyromonas gingivalis minor fimbriae are required for cell-cell interactions. Infect Immun. 2006;74(10):6011-5.

33. Missailidis CG, Umeda JE, Ota-Tsuzuki C, Anzai D, Mayer MP. Distribution of fimA genotypes of Porphyromonas gingivalis in subjects with various periodontal conditions. Oral Microbiol Immunol. 2004;19(4):224-9.

34. Amano A, Nakagawa I, Okahashi N, Hamada N. Variations of Porphyromonas gingivalis fimbriae in relation to microbial pathogenesis. J Periodontal Res. 2004;39(2):136-42.

35. Nakano K, Kuboniwa M, Nakagawa I, Yamamura T, Nomura R, Okahashi $\mathrm{N}$, et al. Comparison of inflammatory changes caused by Porphyromonas gingivalis with distinct fimA, genotypes in a mouse abscess model. Oral Microbiol Immunol. 2004;19(3):205-9.

36. Enersen $M$, Olsen I, Kvalheim $\varnothing$, Caugant DA. fimA genotypes and multilocus sequence types of Porphyromonas gingivalis from patients with periodontitis. J Clin Microbiol. 2008;46(1):31-42.

37. Moreno S, Jaramillo A, Parra B, Botero JE, Contreras A. Porphyromonas gingivalis Fim-A genotype distribution among Colombians. Colomb Med (Cali). 2015;46(3):122-7.

38. Perez-Chaparro PJ, Rouillon A, Minet J, Lafaurie Gl, Bonnaure-Mallet M. fimA genotypes and PFGE profile patterns in Porphyromonas gingivalis isolates from subjects with periodontitis. Oral Microbiol Immunol. 2009;24(5):423-6.

39. Castillo-Rojas G, Cerbón MA, López-Vidal Y. Presence of Helicobacter pylori in a Mexican Pre-Columbian Mummy. BMC Microbiol. 2008:8(1):119.

\section{Publisher's Note}

Springer Nature remains neutral with regard to jurisdictional claims in published maps and institutional affiliations. 